ANL-PHY-7524-TH-93

\title{
Vacuum Structures in Hamiltonian Light-Front Dynamics
}

\author{
F. Coester ${ }^{1}$ and W. Polyzou ${ }^{2}$
}

April 1993

${ }^{1}$ Physics Division, Argonne National Laboratory, Argonne IL 60439

${ }^{2}$ Department of Physics and Astronomy, The University of Iowa, Iowa City, IA 52242

\begin{abstract}
Hamiltonian light-front dynamics of quantum fields may provide a useful approach to systematic non-perturbative approximations to quantum field theories. We investigate inequivalent Hilbert-space representations of the light-front field algebra in which the stability group of the light-front is implemented by unitary transformations. The Hilbert space representation of states is generated by the operator algebra from the vacuum state. There is a large class of vacuum states besides the Fock vacuum which meet all the invariance requirements. The light-front Hamiltonian must annihilate the vacuum and have a positive spectrum. We exhibit relations of the Hamiltonian to the nontrivial vacuum structure.
\end{abstract}




\section{Background}

In the usual formulation of quantum mechanics of finite systems kinematics and dynamics are cleanly separated. An operator algebra of coordinate, momentum and spin operators describes the degrees of freedom of the system. The Hilbert-space representation of this algebra is unique within unitary equivalence. [1] The dynamics is specified by the Hamiltonian operator which is a function of the dynamical variables. Many different dynamical laws exist for any particular Hilbert space of states and the associated operator algebra. In particular states of interacting and noninteracting particles are in the same Hilbert space and the operator algebra is not altered by the dynamics. This picture changes qualitatively for infinite systems. There are inequivalent Hilbert-space representations of the canonical commutation relations. [2] - [5] The Fock-space representation is only one of infinitely many Hilbert-space representations, with different vacuum structure. The vacuum structure depends on the dynamics. The essential ingredients are vacuum polarization terms in the Hamiltonian and invariance under translations. The Lorentz invariance of quantum field theories is not relevant in this context. [6, 7]

In light-front Hamiltonian dynamics [8] there are important differences from the usual equal-time picture. The kinematic symmetries are the transformations which leave the null plane $x^{+}:=t+x_{3}=0$ invariant. The generator, $P^{+}$, of translations tangential to the light cone has a positive continuous spectrum $[0, \infty]$ and a point eigenvalue 0 for the vacuum state. The most important consequence is the suppression of vacuum polarization terms in the Hamiltonian. [9] This feature indicates that a Fock-space representation of quantum field theories may provide a framework for systematic nonperturbative approximations. Discretizations and Tamm-Dancoff truncations of the Fock space have been investigated for that purpose. 10 10 In discretized formulations the operator $P^{+}$has a discrete spectrum. In the expansion of fields modes with $P^{+}=0$, (i.e., "zero-modes") can play an important role. The dynamics generates constraint equations which serve to eliminate these modes. 14 - 17

The standard approach consists of quantization of classical field theories. We believe it is preferable to consider quantum theory as the fundamental theory and construct quantum field theory at the outset as translationally invariant quantum theory. [18, 19] Relativistic quantum mechanics of infinitely 
many free particles is easily formulated in a Hilbert space which is the infinite direct sum of tensor products of one-particle Hilbert spaces. (Fock space) Field operators constructed from particle creation and annihilation operators are precisely the usual free fields and the generators of the Poincaré group obtained in this manner are identical to those obtained by integrating appropriate components of the energy-momentum tensor over the null plane $x^{+}=0$. The Fock vacuum is the only state vector in this Hilbert space which is invariant under translations. The free field Hamiltonian annihilates the vacuum. If a Hamiltonian with interactions also annihilates the vacuum we have the same pattern as in quantum mechanics. The Hilbert space of states and the operator algebra are the same for systems with and without interaction.

From the analogy of field algebras with Euclidean kinematics [7, 20] we expect that there are inequivalent Hilbert space representations of the nullplane field algebra required by classes of Hamiltonians with different properties. In this context it is essential to observe that the construction of field operators described above does not yield operator valued functions of points on the light front but linear functionals (distributions) over suitable test functions defined on the null plane $x^{+}=0$. 21]-23 Important qualitative differences from the Euclidean case are associated with the stability group of the null plane and the distinctive features of the appropriate test functions.

Interactions are introduced formally by adding to the free-field energy momentum tensor an interaction term which can be obtained from a local Lagrangean density. The energy-momentum tensor so constructed is an operator valued functional of functions over the four-dimensional space-time, but it is not an operator functional of functions restricted to the null plane. Thus the formal Hamiltonians obtained from local Lagrangeans are not operators unless they are "regularized". The cut-offs involved in the regularization always destroy the Poincaré covariance, but the null-plane symmetry can be preserved in many ways. Different regularizations may imply different vacuum structures.

The central question is whether there are classes of regularized Hamiltonians which can provide systematic nonperturbative approximations to the observable consequences of the full field theory. Here we will be concerned only with preliminary questions concerning the existence of inequivalent Hilbert space representations of the null-plane field algebra and the associated vacuum structures. It is vital to regularize the Hamiltonians of the local field 
theories in a manner which preserves the nontrivial structure of the physical vacuum.

The purpose of this paper is to exhibit inequivalent Hilbert space representations of the free-field operator algebra and the associated vacuum structures. Invariance and the cluster properties are essential features of these vacuum structures, each of which supports different classes of Hamiltonians. We do not address the questions related to limiting procedures designed to restore Poincaré invariance. Our approach in this paper is exploratory. While the language of algebraic field theory 24] is essential for the exposition of the relevant ideas we do not attempt a mathematically rigorous treatment at this point. The basic assumptions that specify the null-plane field algebra are formulated in Section 2. This is essentially an adaptation of the well-known Wightman axioms [25, 26] and of the basic assumptions of algebraic quantum field theory.[27] The vacuum structures are discussed in Section 3.

\section{Null-Plane Fields}

For any point $x$ in Minkowski space we use the light-front components $x^{+}:=x^{0}+x^{3}, x^{-}:=x^{0}-x^{3}$ and $x_{\perp}=\left\{0, x_{1}, x_{2}, 0\right\}$. For points in the null plane $x^{+}=0$, denoted by $\mathbf{x}:=\left\{x^{-}, x_{\perp}\right\}$, the scalar product $p \cdot x$ reduces to

$$
p \cdot x=p_{\perp} \cdot x_{\perp}-\frac{1}{2} p^{+} x^{-} .
$$

The null-plane component $p^{+}$is positive if $p$ is a timelike four-momentum with positive energy. The null plane $x^{+}=0$ is invariant under a subgroup $\mathcal{N}$ of the Poincaré group which consists of the translations $\mathbf{x} \rightarrow \mathbf{x}+\mathbf{d}$ and the Lorentz transformations $\boldsymbol{\Lambda}(a, b)$ with the $S L(2, C)$ representation

$$
\Lambda(a, b) \rightarrow\left(\begin{array}{cc}
a & 0 \\
b & 1 / a
\end{array}\right)
$$

and the space-time inversion $\mathbf{x} \rightarrow-\mathbf{x}$. We use the notation $\{\mathbf{d}, \boldsymbol{\Lambda}\}$ for the transformation $\mathbf{x} \rightarrow \boldsymbol{\Lambda}(\mathbf{x}+\mathbf{d})$, and for any function $f(\mathbf{x})$

$$
\{\mathbf{d}, \boldsymbol{\Lambda}\} f(\mathbf{x}):=f[\boldsymbol{\Lambda}(\mathbf{x}+\mathbf{d})] .
$$

The group $\mathcal{N}$ is the kinematic subgroup of null-plane dynamics. When the Euclidean group in three dimensions is the kinematic group every hyperplane 
$x^{0}=$ const. is invariant. It is a distinctive feature of null-plane dynamics that only the null plane $x^{+}=0$ is invariant under $\mathcal{N}$. The null planes $x^{+}=$ const. $\neq 0$ are invariant only under a subgroup of $\mathcal{N}$.

\subsection{Polynomial Algebra of Field Operators}

The properties of null-plane fields can be described by a series of assumptions which follow the pattern of the Wightman axioms

\section{A. Assumptions of Null-Plane Quantum Theory}

The states of the theory are described by unit rays in a separable Hilbert space $\mathcal{H}$. The kinematic transformations are given by a continuous unitary representation of the stability group $\mathcal{N}$ of the null plane $\{\mathbf{d}, \boldsymbol{\Lambda}\} \rightarrow U(\mathbf{d}, \boldsymbol{\Lambda})$. Since $U(\mathbf{d}, 1)$ is unitary it can be written as $U(\mathbf{d}, 1)=\exp (i \mathbf{P} \cdot \mathbf{d})$, where $\mathbf{P}:=\left\{P^{+}, P_{\perp}\right\}$ is an unbounded selfadjoint operator interpreted as the light-front momentum operator of the theory. The spectrum of $P^{+}$is positive, $P^{+} \geq 0$. (spectral condition) The infinitesimal generators of the kinematic Lorentz transfor-

mations, $\Lambda$, are $K_{z}$ and $\vec{E}$ for the light-front boosts and $J_{z}$ for the rotations about the longitudinal axis.

There is an invariant state, $\Omega: U(\mathbf{d}, \boldsymbol{\Lambda}) \Omega=\Omega$, unique up to a constant phase factor.

B. Assumptions about the Domain of the Field Operators

1. Fields are "operator valued distributions" over the null plane,

$$
\phi(f):=\int d^{3} \mathbf{x} \phi(\mathbf{x}) f(\mathbf{x})=\int d^{3} \mathbf{p} \phi(-\mathbf{p}) f(\mathbf{p})
$$

where $d^{3} \mathbf{x}:=\frac{1}{2} d x^{-} d^{2} x_{\perp}$ and $d^{3} \mathbf{p}:=d p^{+} d^{2} p_{\perp}$. The test functions $f(\mathbf{x})$ are real valued functions related to the functions $f(\mathbf{p})=$ $\bar{f}(-\mathbf{p})$ by the Fourier transform

$$
\begin{aligned}
& f(\mathbf{x})=(2 \pi)^{-\frac{3}{2}} \int d^{3} \mathbf{p} f(\mathbf{p}) e^{i \mathbf{p} \cdot \mathbf{x}} \\
& f(\mathbf{p})=(2 \pi)^{-\frac{3}{2}} \int d^{3} \mathbf{x} f(\mathbf{x}) e^{-i \mathbf{p} \cdot \mathbf{x}}
\end{aligned}
$$


A suitable test function space $\hat{\mathcal{S}}$ has been defined by Schlieder and Seiler. 21] By their definition $f(\mathbf{x}) \in \hat{\mathcal{S}}\left(\Re^{3}\right)$ are derivatives of functions in the space $\mathcal{S}$ defined by Laurent Schwartz. [22, 23] The functions $f(\mathbf{p}) \in \hat{\mathcal{S}}$ are Schwartz functions in $\mathcal{S}\left(\Re^{3}\right)$ multiplied by $p^{+}$.

2. The operators $\phi(f)$ and their adjoints $\phi^{*}(f)$ are unbounded operators acting on $\mathcal{H}$ defined on some dense domain $\mathcal{D} \subset \mathcal{H}$. The domain $\mathcal{D}$ is a linear set containing the vacuum state $\Omega, \Omega \in \mathcal{D}$. The ranges of operators $U(\mathbf{d}, \boldsymbol{\Lambda}), \phi(f)$ and $\phi(f)^{*}$ are in $\mathcal{D}$,

$$
U(\mathbf{d}, \boldsymbol{\Lambda}) \mathcal{D} \subset \mathcal{D}, \quad \phi(f) \mathcal{D} \subset \mathcal{D}, \quad \phi^{*}(f) \mathcal{D} \subset \mathcal{D} .
$$

If $\Psi_{1}, \Psi_{2} \in \mathcal{D}$, then $\left(\Psi_{1}, \phi(f) \Psi_{2}\right)$ is a linear functional of $f$, which satisfies

$$
\left\langle\Psi_{1} \mid \phi^{*}(f) \Psi_{2}\right\rangle=\overline{\left\langle\Psi_{2} \mid \phi(f) \Psi_{1}\right\rangle} .
$$

3. In general there will be several types of fields (distinguished by an index $i$ ) each with tensor or spinor components (labeled by an index $\lambda$ ). We have then

$$
\phi(f):=\sum_{i} \int d^{3} \mathbf{x} \phi_{\lambda}^{i}(\mathbf{x}) f^{i \lambda}(\mathbf{x}) .
$$

C. Transformation Law of the Fields

The fields transform according to

$$
U^{-1}(\mathbf{d}, \boldsymbol{\Lambda}) \phi_{\lambda}^{i}(\mathbf{x}) U(\mathbf{d}, \boldsymbol{\Lambda})=S_{\lambda}^{(i) \rho}(\boldsymbol{\Lambda}) \phi_{\rho}^{i}\left(\boldsymbol{\Lambda}^{-1} \mathbf{x}-\mathbf{d}\right),
$$

where $S_{\lambda}^{(i) \rho}(\boldsymbol{\Lambda})$ is a finite dimensional representation matrix of the Lorentz transformation $\boldsymbol{\Lambda}$.

D. Canonical Commutation relations, Local Commutativity

Charged scalar fields satisfy the canonical commutation relations,

$$
\left[\phi(f), \phi^{*}(g)\right]=(f, g)-(g, f), \quad[\phi(f), \phi(g)]=0,
$$

where the scalar product of the test functions is defined by

$$
(f, g):=\int \frac{d^{3} \mathbf{p} \theta\left(p^{+}\right)}{p^{+}} \bar{f}(\mathbf{p}) g(\mathbf{p}) .
$$


If $\phi(f)$ is a spinor field we have the corresponding anticommutation relations

$$
\left[\phi(f), \phi^{\dagger}(g)\right]_{+}=(f, g)+(g, f), \quad[\phi(f), \phi(g)]_{+}=0
$$

where the invariant inner product of the spinor test functions is

$$
(f, g):=\int \frac{d^{3} \mathbf{p} \theta\left(p^{+}\right)}{p^{+}} f^{\dagger}(\mathbf{p}) g(\mathbf{p}) .
$$

Here $\phi^{\dagger}(g):=\phi^{*}(g) \beta$ and $f^{\dagger}(\mathbf{p}):=\bar{f}(\mathbf{p}) \beta$ and $\beta \equiv \gamma^{0}$ is the usual Dirac matrix.

For Hermitean scalar fields, $\phi^{*}(f)=\phi(f)$, we have,

$$
[\phi(f), \phi(g)]=\frac{1}{2}[(f, g)-(g, f)] \text {. }
$$

or

$$
\left[\phi(\mathbf{p}), \phi\left(\mathbf{p}^{\prime}\right)\right]=\frac{\delta\left(\mathbf{p}+\mathbf{p}^{\prime}\right)}{2 p^{+}}
$$

Two points, $\mathbf{x}$ and $\mathbf{x}^{\prime}$, on the null plane are relatively spacelike if $\left|x_{\perp}-x_{\perp}^{\prime}\right|>0$. The scalar product $(f, g)$ vanishes when the supports of the test functions $f$ and $g$ are relatively spacelike.

\section{E. Completeness}

Polynomials of the operators $\phi(f)$ operating on the vacuum state $\Omega$ generate a dense set of states in $\mathcal{H}$.

\section{F. Dynamics}

There exists a self-adjoint Hamiltonian operator $H \equiv P^{-}$whose domain contains the domain $\mathcal{D}$ of Assumption B. The Hamiltonian operator $P^{-}$ is invariant under the null-plane translations, $\left[P^{-}, \mathbf{P}\right]=0$. The operator $P^{-}$together with the generators, $\mathbf{P}$, of the null-plane translations transforms as a four-vector under the null-plane Lorentz transformations (2.2). The mass operator, $M^{2}:=P^{+} P^{-}-P_{\perp}^{2}$ is invariant and has a positive spectrum. The spectrum of the Hamiltonian is the positive real axis. The Hamiltonian operator annihilates the vacuum. 
The following discussions will be restricted throughout to Hermitean scalar fields satisfying the commutation relations (2.14). We assume that the operator algebra is not modified by the dynamics. With the definitions

$$
\phi^{( \pm)}(f):=\int d^{3} \mathbf{p} \phi^{( \pm)}(\mathbf{p}) f(-\mathbf{p}), \quad \phi^{( \pm)}(\mathbf{p}):=\theta\left( \pm p^{+}\right) \phi(\mathbf{p})
$$

it follows from the commutation relations (2.15) and the definition of the test function space that

$$
\left[\phi^{(+)}(f), \phi^{(+)}(g)\right]=\left[\phi^{(-)}(f), \phi^{(-)}(g)\right]=0
$$

and

$$
\left[\phi^{(+)}(f), \phi^{(-)}(g)\right]=\frac{1}{2}(f, g) .
$$

We can therefore define the "normal ordered product" of field operators in the usual manner, e.g.,

$$
\begin{aligned}
: \phi\left(f_{1}\right) \phi\left(f_{2}\right): & :=\phi^{(+)}\left(f_{1}\right) \phi^{(+)}\left(f_{2}\right)+\phi^{(-)}\left(f_{1}\right) \phi^{(-)}\left(f_{2}\right) \\
& +\phi^{(-)}\left(f_{1}\right) \phi^{(+)}\left(f_{2}\right)+\phi^{(-)}\left(f_{2}\right) \phi^{(+)}\left(f_{1}\right) .
\end{aligned}
$$

From the kinematic covariance of the field and the spectral condition it follows that

$$
\left[P^{+}, \phi(f)\right] \Omega=\int d^{3} \mathbf{p} p^{+} \theta\left(p^{+}\right) \phi(-\mathbf{p}) f(\mathbf{p}) \Omega
$$

and hence

$$
\left[P^{+}, \phi^{(+)}(f)\right] \Omega=\int d^{3} \mathbf{p} p^{+} \theta\left(p^{+}\right) \phi(\mathbf{p}) f(-\mathbf{p}) \Omega=0, \quad \frac{\partial \phi^{(+)}(\mathbf{x})}{\partial x^{-}} \Omega=0 .
$$

The operators $\phi^{(+)}(f)$ annihilate the vacuum for all test functions $f(\mathbf{p})$ whose support excludes some neighborhood of $p^{+}=0$. If we assume that $\phi^{(+)}(f)$ annihilates the vacuum for all $f$ then the vacuum is the Fock vacuum and we have the usual Fock-space representation of the field algebra. Any nontrivial vacuum structure must be associated with vanishing values of $p^{+}$.

The generators of the light-front symmetries are obtained from integrals over the light-front components of the energy momentum tensor,

$$
P^{+}=\int d^{3} \mathbf{x} T^{++}(\mathbf{x}), \quad P_{\perp}=\int d^{3} \mathbf{x} T^{+\perp}(\mathbf{x})
$$




$$
\begin{aligned}
& K_{3}=\int d^{3} \mathbf{x} T^{++}(\mathbf{x}) x^{-}, \quad \vec{E}=\int d^{3} \mathbf{x} T^{++}(\mathbf{x}) x_{\perp} \\
& J_{3}=\int d^{3} \mathbf{x}\left[T^{+2}(\mathbf{x}) x_{1}-T^{+1}(\mathbf{x}) x_{2}\right],
\end{aligned}
$$

where

$$
T^{++}(\mathbf{x})=4: \frac{\partial \phi(\mathbf{x})}{\partial x^{-}} \frac{\partial \phi(\mathbf{x})}{\partial x^{-}}:
$$

and

$$
T^{+\perp}(\mathbf{x})=2: \frac{\partial \phi(\mathbf{x})}{\partial x_{\perp}} \frac{\partial \phi(\mathbf{x})}{\partial x^{-}}:
$$

The kinematic generators annihilate vacuum even when there is a nontrivial vacuum structure at $p^{+}=0$.

According to Assumption $\mathrm{F}$ we have

$$
P^{-}=\frac{M^{2}+P_{\perp}^{2}}{P^{+}}
$$

and in analogy to $(2.21)$

$$
\left[P^{-}, \phi^{(+)}(f)\right] \Omega=P^{-} \phi^{(+)}(f) \Omega=0 .
$$

For the free-field Hamiltonian,

$$
P^{-}=\int d^{3} \mathbf{x} T^{+-}(\mathbf{x})=\int d^{3} \mathbf{x}: \phi(\mathbf{x})\left[m^{2}-\nabla_{\perp}^{2}\right] \phi(\mathbf{x}):
$$

we get from eq. (2.26)

$$
\left[P^{-}, \phi^{(+)}(f)\right] \Omega=\int d^{3} \mathbf{p} \frac{m^{2}+p_{\perp}^{2}}{p^{+}} \theta\left(p^{+}\right) \phi(\mathbf{p}) f(-\mathbf{p}) \Omega=0 .
$$

It follows that the free-field vacuum is the Fock vacuum, which by definition is annihilated by $\phi^{(+)}(f)$ for all $f$. In general the condition (2.26) may specify annihilation operators which require a zero-mode structure of the vacuum. A similar characterization of the vacuum structure by the annihilation operators was found in the Euclidean case [6].

The most convenient description of general vacuum structures results from the formulation of the field algebra as an abstract *-algebra[27] described in the next subsection. 


\subsection{Abstract *-Algebra}

The properties of field operators listed above can be derived from the abstract *-algebra generated by finite linear combinations,

$$
R:=\sum_{n} c_{n} U\left(f_{n}\right)
$$

with complex coefficients $c_{n}$ and Schlieder-Seiler test functions $f_{n}(\mathbf{x})$. Here the unitaries $U(f)$ have the algebraic properties and the norm topology of linear combinations of the Hilbert-space operators $\exp [i \phi(f)]$ considered in the previous subsection. We have thus a $C^{*}$ algebra,

$$
\left\|R^{*} R\right\|=\|R\|^{2}
$$

with the following properties:

I. Involution

$$
R^{*}=\left[\sum_{n} c_{n} U\left(f_{n}\right)\right]^{*}=\sum_{n} \bar{c}_{n} U\left(-f_{n}\right) .
$$

II. Product and Unit Element

$$
U(g) U(f)=U(f+g) e^{\frac{1}{4}[(f, g)-(g, f)]}, \quad U(0)=1 .
$$

With the notation $U\left(f_{1}, f_{2}\right):=U(f)$, where $f(\mathbf{p})=f_{1}(\mathbf{p})-i f_{2}(\mathbf{p})$ and $\left.f_{(} \mathbf{p}\right), f_{2}(\mathbf{p})$ are real, we see the the algebra specified by eq. (2.32) is the Weyl algebra, 28

$$
U\left(g_{1}, g_{2}\right) U\left(f_{1}, f_{2}\right)=U\left(g_{1}+f_{1}, g_{2}+f_{2}\right) e^{\frac{i}{2}\left[\left(f_{2}, g_{1}\right)-\left(f_{1}, g_{2}\right)\right]} .
$$

\section{Null-Plane Symmetry}

The continuous null-plane symmetries are implemented by *-automorphisms of the abstract algebra,

$$
\{\mathbf{d}, \boldsymbol{\Lambda}\} U(f) \rightarrow U(\{\mathbf{d}, \boldsymbol{\Lambda}\} f),
$$

where

$$
\{\mathbf{d}, \boldsymbol{\Lambda}\} f(\mathbf{x})=f[\boldsymbol{\Lambda}(\mathbf{x}+\mathbf{d})] .
$$

The space-time inversion $\mathrm{x} \rightarrow-\mathbf{x}$ is implemented by the anti-automorphism

$$
c U(f) \rightarrow \bar{c} U(-\tilde{f})
$$

where $\tilde{f}(\mathbf{x}):=f(-\mathbf{x})$. 


\section{The Vacuum Functional}

The vacuum state is given by a continuous linear functional over the algebra, $E[R]=\sum_{i} c_{i} E\left[U\left(f_{i}\right)\right], E(f):=E[U(f)]$, with the properties:

1. Positivity: $E\left[R^{*} R\right]=\sum c_{n} \bar{c}_{m} E\left(f_{n}-f_{m}\right) e^{\frac{1}{4}\left[\left(f_{n}, f_{m}\right)-\left(f_{m}, f_{n}\right)\right]} \geq 0$.

2. Normalization: $E(0)=E[1]=1$.

3. Reality: $\bar{E}(f)=E(-f)$

4. Invariance: $E(f)=E(\{\mathbf{d}, \boldsymbol{\Lambda}\} f), \quad E(f)=E(\tilde{f})$

5. Cluster Property: $\lim _{\lambda \rightarrow \infty}[E(f+\{\lambda \mathbf{d}\} g)-E(f) E(g)]=0, d_{\perp} \neq 0$.

For a vacuum functional with these properties the Gelfand-Naimark-Segal (GNS) construction [27] provides a Hilbert-space representation of the algebra:

$$
\langle\Omega \mid R \Omega\rangle=E[R],
$$

and for any two state vectors $\Psi_{1}:=R_{1} \Omega, \Psi_{2}:=R_{2} \Omega$

$$
\left\langle\Psi_{2} \mid \Psi_{1}\right\rangle=E\left[R_{2}^{*} R_{1}\right] .
$$

There is only one translationally invariant vector, $\Omega$, in the Hilbert space so constructed. (See ref. [7] theorem 6.1)

\section{Vacuum Structure}

From the strong continuity of the operators $U(f)$ follows the existence of self-adjoint field operators $\phi(f)$ such that $U(f)=e^{i \phi(f)}$. The explicit form of the Fock vacuum functional,

$$
E_{0}(f):=e^{-\frac{1}{4}(f, f)}
$$

follows immediately from the normal ordered form

$$
U(f)=e^{i \phi^{(-)}(f)} e^{i \phi^{(+)}(f)} e^{-\frac{1}{4}(f, f)},
$$

which is a consequence of the commutation relations (2.18) and the BakerCampbell-Hausdorff formula [29]. 
The vacuum functional determines the Hilbert space representation of the operator algebra. The existence of nontrivial Hilbert representations depends on invariant functionals of the test functions $f(\mathbf{p})$ which depend only on the neighborhood of $p^{+}=0$. Examples are functionals of

$$
f_{0}\left(p_{\perp}\right):=\lim _{p^{+} \rightarrow 0} \frac{f(\mathbf{p})}{i p^{+}}
$$

for instance $f_{0}(0)$ and

$$
\int d^{2} p_{\perp}\left|f_{0}\left(p_{\perp}\right)\right|^{2} \eta\left(\left|p_{\perp}\right|^{2}\right)
$$

where $\eta\left(\left|p_{\perp}\right|^{2}\right)$ is some fixed positive function.

A dense set of states can be represented by sets of functions $\psi^{(n)}\left(\mathbf{x}_{1}, \ldots \mathbf{x}_{n}\right)$ in the $n$-fold tensor product of $\hat{\mathcal{S}}\left(\Re^{3}\right)$, because the states

$$
\Psi:=\sum_{n} \int d^{3} \mathbf{x}_{1} \ldots \int d^{3} \mathbf{x}_{n} \phi\left(\mathbf{x}_{1}\right) \ldots \phi\left(\mathbf{x}_{n}\right) \Omega \psi^{(n)}\left(\mathbf{x}_{1}, \ldots \mathbf{x}_{n}\right)
$$

are dense in $\mathcal{H}$. The scalar product $\left\langle\Psi_{2} \mid \Psi_{1}\right\rangle$ of the states $\Psi_{2}$ and $\Psi_{1}$ is therefore related to the vacuum distribution

$$
W_{n}\left(\mathbf{x}_{1}, \ldots, \mathbf{x}_{n}\right):=E\left[\phi\left(\mathbf{x}_{1}\right) \cdots \phi\left(\mathbf{x}_{n}\right)\right]
$$

by

$$
\begin{aligned}
& \left\langle\Psi_{2} \mid \Psi_{1}\right\rangle=\sum_{n, m} \int d^{3} \mathbf{x}_{1} \cdots \int d^{3} \mathbf{x}_{n+m} \\
& \times \bar{\psi}_{2}^{(n)}\left(\mathbf{x}_{1}, \ldots, \mathbf{x}_{n}\right) W_{n+m}\left(\mathbf{x}_{1}, \ldots, \mathbf{x}_{n+m}\right) \psi_{1}^{m}\left(\mathbf{x}_{n+1}, \ldots, \mathbf{x}_{n+m}\right) .
\end{aligned}
$$

The right-hand side of eq. (3.7) is a positive invariant inner product of functions in $\oplus_{n} \hat{\mathcal{S}}^{\otimes n}$. We have by completion a realization of the Hilbert space by direct sums of multi-point functions. The inner product of the functions representing states is determined by the vacuum distributions.

Nontrivial vacuum structures become apparent when we consider the vacuum expectation values of the normal ordered operator 


$$
\begin{aligned}
& : U(f):=e^{i \phi^{(-)}(f)} e^{i \phi^{(+)}(f)}=U(f) e^{\frac{1}{4}(f, f)} \\
& =\sum_{n} \frac{i^{n}}{n !} \int d^{3} \mathbf{x}_{1} \cdots \int d^{3} \mathbf{x}_{n}: \phi\left(\mathbf{x}_{1}\right) \cdots \phi\left(\mathbf{x}_{n}\right): f\left(\mathbf{x}_{1}\right) \cdots f\left(\mathbf{x}_{n}\right) .
\end{aligned}
$$

The cluster structure of the vacuum requires that the normal ordered vacuum distributions

$$
w_{n}\left(\mathbf{x}_{1}, \ldots, \mathbf{x}_{n}\right):=E\left[: \phi\left(\mathbf{x}_{1}\right) \cdots \phi\left(\mathbf{x}_{n}\right):\right]
$$

are products of "truncated vacuum distributions" [30, $w_{T}^{(n)}\left(\mathbf{x}_{1}, \ldots, \mathbf{x}_{n}\right)$, which vanish in the limit of large spacelike separations of any two points. They can be defined by

$$
E[: U(f):]=\exp \left[\sum_{n} i^{n} w_{T}^{(n)}(f)\right],
$$

where

$$
w_{T}^{(n)}(f):=\frac{1}{n !} \int d^{3} \mathbf{x}_{1} \cdots \int d^{3} \mathbf{x}_{n} w_{T}^{(n)}\left(\mathbf{x}_{1}, \ldots, \mathbf{x}_{n}\right) f\left(\mathbf{x}_{1}\right) \cdots f\left(\mathbf{x}_{n}\right) .
$$

The distributions (3.9) can be obtained from (3.10) by functional differentiation.

Nonvanishing distributions $w_{T}^{(n)}(f)$ represent nontrivial vacuum structure. The requirement (2.21) that the operator $\left[P^{+}, \phi(f)\right]$ annihilates the vacuum in all representations is satisfied when the vacuum distributions $w_{T}^{(n)}(f)$ involve only the restriction (3.3).

$$
\begin{aligned}
w_{T}^{(n)}(f) & :=\frac{1}{n !} \int d^{3} \mathbf{p}_{1} \ldots \int d^{3} \mathbf{p}_{n} \hat{w}_{T}^{(n)}\left(p_{1 \perp}, \ldots, p_{n \perp}\right) \delta\left(p_{1 \perp}+\ldots+p_{n \perp}\right) \\
& \times \frac{\delta\left(p_{1}^{+}\right)}{i p^{+}} f\left(\mathbf{p}_{1}\right) \cdots \frac{\delta\left(p_{1}^{+}\right)}{i p^{+}} f\left(\mathbf{p}_{n}\right)
\end{aligned}
$$

These vacuum structures are formally the same as those of a nonrelativistic Bose gase in two space dimensions. [20] The positivity of the spectrum of the Hamiltonian $P^{-}$together with the condition that the Hamiltonian must annihilate the vacuum led to the requirement $(2.26),\left[P^{-}, \phi^{(+)}(f)\right] \Omega=0$. 
This requirement relates the dynamics specified by the Hamiltonian to a particular vacuum structure.

Simple examples of a nontrivial vacuum are obtained when only $w_{T}^{(1)}(f)$ and/or $w_{T}^{(2)}(f)$ are different from zero, for instance,

$$
w_{T}^{(1)}(f)=\varphi_{0} f_{0}(0),
$$

for some real parameter $\varphi_{0}$, and

$$
w_{T}^{(2)}(f)=\frac{1}{2} \int d^{2} p_{\perp}\left|f_{0}\left(p_{\perp}\right)\right|^{2} \eta\left(\left|p_{\perp}\right|^{2}\right) .
$$

for some fixed function $\eta$. Such simple Gaussian vacua may be the basis of non-Fock perturbative approximations.

\section{Summary and Conclusions}

A superficial inspection of the Fock-space formalism indicates that the Fock vacuum is not a stationary state when there are pure creation terms in the normal ordered interaction Hamiltonian. In an equal-time Hamiltonian formalism such terms are always present when the Hamiltonians are derived from local Lagrangeans. Since the Fock vacuum is the only translationally invariant state vector in the Fock space, the physical vacuum is the cyclic vector of an inequivalent Hilbert-space representation of the field algebra.

In light-front Hamiltonian dynamics it appears superficially that pure creation terms in the Hamiltonian cannot exist and that the Fock vacuum is therefore the physical vacuum. This observation rests on the requirement that the sum $p_{1}^{+}+\ldots+p_{n}^{+}$vanishes which implies that each individual $p_{i}^{+}$ vanishes. For a more careful analysis it is essential to note that the fields are defined as distributions over Schlieder-Seiler test functions and that $\delta\left(p^{+}\right) / p^{+}$ is well defined as a distribution. It follows that nontrivial vacuum structures exist also in Hamiltonian light-front dynamics. When a light-front Hamiltonian that is derived formally from a Lagrangean always possible to suppress the pure creation terms without affecting the null-plane invariance. It remains to be seen what if any role the vacuum structures play in approximations to Poincaré invariant field theories. The role of quark condensates may give an indication. They can be accommodated in this framework, but only in the presence of a nontrivial vacuum. 


\section{Acknowledgments}

This work was supported by the Department of Energy, Nuclear Physics

Division, under contracts W-31-109-ENG-38 (F.C.) and DE-FG02-86ER40286 (W.P.)

\section{References}

[1] N. N. Bogolubov et al. General Principles of Quantum Theory (Kluwer Academic Publishers, Dodrecht 1990). See page 262.

[2] L. Van Hove, Physics, 18 (1952) 145.

[3] K. O. Friedrics, Mathematical Aspects of the Quantum Theory of Fields. (Interscience Pulbl., New York 1953).

[4] L. Gårding, and A. S. Wightman, Proc. Nat. Acad. 40 (1956) 622.

[5] I. E. Segal, Trans. Am. Math. Soc. 88 (1958) 12.

[6] F. Coester and R. Haag, Phys. Rev., 117 (1960) 1137.

[7] H. Araki, J. Math. Phys., 1 (1964),492.

[8] P.A.M. Dirac, Rev. Mod. Phys.21 (1949) 329 .

[9] S. Weinberg, Phys. Rev. 150 (1966) 1313.

[10] H. C. Pauli and S. J. Brodsky, Phys. Rev. D32 (1985) 1993; 2001.

[11] R. J. Perry, et al. Phys. Rev. Lett. 65 (1990) 2959.

[12] D. Mustaki et al. Phys. Rev. D43 (1991) 3411.

[13] A. C. Tang et al. Phys. Rev.D44 (1991) 1842.

[14] T. Maskawa and K Yamawaki, Prog. Theor. Phys. 56 (1976) 270.

[15] Th. Heinzl et al. Z. Phys. A334 (1989) 443; Phys. Lett. B 256 (1991); Nuc. Phys. A532 (1991) 429c; Regensburg Preprint TPR 92-17. 
[16] G. McCartor and D. Robertson, Z. Phys C53 (1992) 679.

[17] S. Pinsky, Ohio State Preprint, OHSTPY-HEP-TH-92-030

[18] F. Rohrlich, Acta Phys. Austriaca, Supp. 8 (1971) 277. See Sec. 5

[19] F. Coester, Prog. Nuc. Part. Phys. 29 (1992) 1.

[20] H. Araki and E. J. Woods, J. Math. Phys.,4 (1963) 637.

[21] S. Schlieder and E. Seiler, Comm. Math. Phys 25 (1972) 62.

[22] L. Schwartz, Théorie des Distributions, (Hermann, Paris 1957)

[23] I. M. Gelfand and G. E. Shilov, Generalized Functions, (Academic Press, New York 1964).

[24] R. Haag, Local Quantum Physics, (Springer 1992).

[25] R. F. Streater and A.S. Wightman, PCT, Spin \& Statistics and All That, (Benjamin, New York 1964).

[26] See Sec. II.1.2 of ref. 24.

[27] See Chapter III of ref. 24]

[28] H. Leutwyler,J. R. Klauder and L. Streit, Nuov. Cim. 66A (1970) 536.

[29] N. Bourbaki, Lie Groups and Lie Algebras, (Springer 1989). See page 160-161.

[30] See Sec. II.2.2 of ref. 24] 